\title{
A propos de deux épidémies de teigne à Sabouraudites (Microsporum) langeroni \\ Vanbreuseghem, 1950, en milieu scolaire français
}

\author{
par B. GILOT (*), M. GALLIEN et G. LEGEAIS \\ Laboratoire de Parasitologie. U.E.R. «santé dans la collectivité » \\ Faculté de Médecine, F 35000 Rennes
}

\begin{abstract}
Résumé
Les auteurs décrivent deux petites épidémies de teigne scolaire dues à un dermatophyte africain très proche de Microsporum audouini : Microsporum langeroni Vanbreuseghem, 1950. C'est la première fois, semble-t-il, que de telles épidémies sont signalées en France.

Après quelques considérations d'ordre clinique et thérapeutique, ils étudient les caractères même de l'épidémie, et essaient d'en expliquer la génèse.

Enfin, ils insistent sur les sanctions d'ordre prophylactique qui en découlent.
\end{abstract}

\section{Summary}

About two epidemies of scalp ringworm in French schools occasioned by Sabouraudites (Microsporum) langeroni Vanbreuseghem, 1950.

The authors describe two minor epidemics of scalp ringworm in schools, caused by an African dermatophyte very similar to

* Attaché de recherche à 1'I.N.S.E.R.M. 


\begin{abstract}
Microsporum audouini: Microsporum langeroni Vanbreuseghem, 1950. This is apparently the first time that such epidemics have been observed in France.

After some reflexions of a clinical and therapeutic nature, they study the actual characteristics of the epidemic and attempt to explain its genesis.

Finally, they insist on measures to be taken on prophylactic grounds.
\end{abstract}

Depuis la fin de la dernière guerre mondiale, il semble que le nombre de cas de teignes microsporiques d'origine humaine ait beaucoup diminué. Dans la région lyonnaise, par exemple, les teignes à $M$. audouini semblent rares par rapport aux teignes à $M$. canis et, à part une épidémie retentissante relatée en 1964 par J. Coudert et al., tout au plus n'est-il signalé, ici ou là, que des cas sporadiques. A Paris, cependant, Badillet et al. rapportent tout récemment une recrudescence du nombre de cas de teignes à $M$. audouini «dans sa variété africaine», mais presque tous les cas cités sont des cas d'importation.

A Grenoble même, cinq années de pratique mycologique n'avaient pas permis de déceler un seul cas de teigne à $M$. audouini. Or, au cours des deux dernières années, 1971 et 1972, nous avons eu l'occasion d'étudier, dans la même école de cette ville, deux petites épidémies de teigne dues à un dermatophyte très proche, morphologiquement, de Microsporum audouini (Gruby, 1843), épidémies dont les caractères nous ont paru assez particuliers pour être rapportés ici, cette étude présentant à nos yeux deux types d'intérêt complémentaires: un intérêt spéculatif et un intérêt d'ordre pratique.

\title{
I. Exposé des faits
}

En 1971, quatre examens mycologiques, effectués de janvier à mars dans une même école de Grenoble, permettent d'isoler des souches très semblables à tout point de vue, que nous avons pu rapprocher de $M$. audouini. En outre, deux enfants teigneux de cette même école ont bénéficié d'un traitement avant même tout prélèvement. Une enquête plus approfondie est décidée, qui ne peut être réalisée que deux mois plus tard. A l'école maternelle, dont font partie cinq des enfants atteints, près de trois cents enfants sont examinés en lumière de Wood. Trois nouveaux cas de teigne à $M$. audouini sont détectés. Deux enfants présentent même une teigne mixte à $M$. audouini et Langeronia soudanensis (Joyeux, 1912). Une seule classe de l'école primaire bénéficie du même examen : aucun cas nouveau n'est décelé.

En 1972, malgré la désinfection effectuée l'année précédente, un nouveau cas avec confirmation mycologique fait entreprendre une nouvelle enquête; on détecte ainsi : un nouveau cas de teigne dû, en fait, à $M$. canis; un volumineux herpès circiné 
frontal, chez une fillette, qui relève de $M$. audouini. En outre, la trace pigmentée de volumineux herpès circinés des membres est retrouvée chez une fillette traitée sans prélèvement.

Au total, entre les deux années, neuf cas relèvent avec certitude de $M$. audouini (sensu lato) et trois cas peuvent être imputés avec vraisemblance au même champignon.

Le tableau I indique la répartition par classe, l'âge, le sexe et l'origine géographique des enfants atteints.

\section{Tableau I}

Etat civil et répartition par classe des enfants teigneux

\begin{tabular}{|c|c|c|c|c|c|c|c|c|}
\hline \multirow{2}{*}{ Nom } & \multirow{2}{*}{ Age } & \multirow{2}{*}{ Sexe } & \multirow{2}{*}{$\begin{array}{l}\text { Origine } \\
\text { géogra- } \\
\text { phique }\end{array}$} & \multicolumn{3}{|c|}{$\begin{array}{l}\text { Nom } \\
\text { de l'institutrice }\end{array}$} & \multirow{2}{*}{ Année } & \multirow{2}{*}{$\begin{array}{l}\text { Preuve } \\
\text { myco- } \\
\text { logique }\end{array}$} \\
\hline & & & & $\begin{array}{r}\text { mater } \\
\text { nelle }\end{array}$ & er- & primaire & & \\
\hline B. Paolo .... & 3 ans $1 / 2$ & M & Portugal & $\mathbf{M}^{\mathrm{me}}$ & Gi. & & 1971 & + \\
\hline $\begin{array}{l}\text { Di. Anne- } \\
\text { Marie } \ldots \ldots \ldots\end{array}$ & 3 ans $1 / 2$ & $\mathrm{~F}$ & Sénégal & 》 & $\gg$ & & 1971 & + \\
\hline E. François .. & 3 ans $1 / 2$ & M & $\begin{array}{l}\text { Came- } \\
\text { roun }\end{array}$ & $\gg$ & $\gg$ & & 1971 & \\
\hline T. Serge $\ldots$. & 5 ans & M & $\begin{array}{l}\text { Came- } \\
\text { roun }\end{array}$ & $M^{m e}$ & $\mathrm{R}$. & & 1971 & + \\
\hline D. Nathalie ... & 5 ans $1 / 2$ & $\mathrm{~F}$ & France & » & $\gg$ & & 1971 & + \\
\hline B. Alain $\ldots$. & 5 ans $1 / 2$ & M & France & 》 & $\gg$ & & 1971 & + \\
\hline $\begin{array}{l}\text { Di. Marie- } \\
\text { Antoinette ... }\end{array}$ & 5 ans $1 / 2$ & $\mathrm{~F}$ & Sénégal & 》 & $》$ & & 1971 & + \\
\hline E. Louis .... & 4 ans $1 / 2$ & M & $\begin{array}{l}\text { Came- } \\
\text { roun }\end{array}$ & $\mathbf{M}^{\mathrm{me}}$ & Ge. & & 1971 & \\
\hline B. Jean-Luc . & 8 ans & M & France & & & $\mathbf{M}^{\mathrm{me}} \mathrm{N}$. & 1971 & + \\
\hline A. Brice $\ldots$. & 3 ans $1 / 2$ & $\mathrm{~F}$ & Gabon & $\mathrm{M}^{\mathrm{me}}$ & Gi. & & 1972 & + \\
\hline B. Noriane .. & 3 ans $1 / 2$ & F & France & $\gg$ & $\gg$ & & 1972 & + \\
\hline P. Frédérique & $?$ & F & France & » & $\gg$ & & 1972 & + \\
\hline
\end{tabular}

$$
\begin{aligned}
& \mathrm{M}=\text { masculin. } \\
& \mathrm{F}=\text { féminin. }
\end{aligned}
$$

Nous décrirons d'abord sommairement les caractéristiques cliniques de ces teignes et nous en préciserons l'évolution sous thérapeutique. Puis, nous essaierons de cerner le problème du point de vue écologique et épidémiologique. 


\section{Données cliniques et thérapeutiques}

\section{A) Caractéristiques cliniques.}

Ce sont des teignes du cuir chevelu que nous avons pu observer dans la plupart des cas et, plus exceptionnellement, des herpès circinés.

\section{LES TEIGNES DU CUIR CHEVELU.}

Deux aspects très différents peuvent être réalisés (parfois il existe un panachage de ces deux aspects). Il s'agit, le plus souvent, de plaques d'assez grande taille (de un à cinq centimètres de diamètre), unique ou multiples (le plus souvent au nombre de deux : trois cas). Cet aspect évoque celui de la teigne miscrosporique à $M$. canis. Cependant, quelques cheveux au centre même de la lésion sont engainés de spores sans être cassés.

Les localisations sont très variées (frontale, temporale, occipitale...).

Les cheveux atteints sont vivement fluorescents en lumière de Wood (d'une fluorescence qui nous a paru plus vive et plus bleutée que celle qu'on observe avec $M$. canis) ;

- il s'agit, parfois, d'un aspect véritablement trichophytique. Cet aspect a été noté chez deux petites sénégalaises dont la teigne a été dépistée au cours de notre enquête de 1971. Dans ces deux cas, les lésions étaient distribuées en pluie sur tout le cuir chevelu. En lumière de Wood, toute la tête nous est apparue fluorescente tant les plaques étaient multiples. Or, les fillettes en question n'avaient même pas été soupçonnées par leur entourage, leur teigne étant pratiquement invisible sans l'aide de la lumière de Wood. Les plaques, de très petite taille, étaient par surcroît masquées par de multiples petites tresses qui constituent un type traditionnel de coiffure pour l'ethnie de ces petites sénégalaises. Ce type très particulier d'atteinte dermatophytique chez les fillettes coiffées avec des tresses (qu'on ne défait pas tous les jours et pour cause...) a été observé, précisément au Sénégal, par Juminer (communication personnelle) ;

- enfin, un aspect mixte peut être réalisé, combinant ces deux formes cliniques : c'est le cas du petit T... Serge dont le cuir chevelu présentait déjà deux plaques temporales bien circonscrites (quatre centimètres et un centimètre cinq de diamètre) et dont, par ailleurs, toute la nuque était fluorescente.

\section{LES HERPÈS CIRCINÉS.}

Un herpès circiné frontal a été noté chez une fillette en 1972: très volumineux (cinq centimètres de diamètre), il présentait un double contour remarquable : cet aspect, assez différent de celui qu'on observe fréquemment avec $M$. canis, n'est pas spécial à M. audouini.

Sans doute faut-il rapporter également à $M$. audouini ces deux séquelles pigmentées de grande taille (six centimètres de diamètre), que nous avons constatées sur les jambes de la petite P... Frédérique, également en 1972. D'autre part, l'interrogatoire nous a appris que l'épidémie de 1971 aurait été précédée d'une efflorescence d'herpès circinés de petite taille sur le visage et les membres inférieurs, précisément dans les classes de maternelle qui seront atteintes en 1971 et 1972 (classes de $\mathbf{M}^{\mathrm{me}} \mathrm{R} \ldots$ et Ge...). Il n'y avait pas apparemment de lésions du cuir chevelu concomitante. 


\section{B) La thérapeutique.}

Dans tous les cas, l'évolution sous thérapeutique a été satisfaisante. C'est une thérapeutique classique qui a été effectuée, associant un traitement par voie générale (Griséfuline : 3 à 4 comprimés à $125 \mathrm{mg}$ pendant 20 jours) à un colmatage local par diverses pommades antifongiques (Thérops, Léofungine, Pimafucine). La guérison définitive a pratiquement toujours été obtenue dans ce laps de temps. Deux fois, cependant, le traitement, pour obtenir une stérilisation complète, a dû être poursuivi quinze jours de plus aux mêmes doses.

\section{Données épidémiologiques}

\section{A) Caractères généraux de l'épidémie.}

a) Il s'agit d'une épidémie de caractère limité (1). Les enfants de la maternelle (environ 300) sont répartis dans deux bâtiments séparés, qui ne communiquent pas entre eux et dont les enfants ne se fréquentent pas sur les cours de récréation. Or les cas de teigne ont été décelés dans un seul bâtiment. Par contre, dans ce bâtiment, trois des quatre classes sont atteintes en 1971, une seule en 1972.

b) L'évolution de l'épidémie de 1971 a été particulièrement lente : si les premiers cas ont été décelés en décembre 1970, l'enquête n'a pu être réalisée qu'en mai 1971. Or le nombre total des enfants atteints en 1971 ne paraît guère dépasser neuf, si même il atteint ce chiffre. Voilà qui contraste singulièrement avec les propos de Sabouraud, combien de fois vérifiés: «La contagiosité de cette maladie, en France du moins, est extrême ; où la microsporie fait-elle le plus de ravages, c'est à l'école primaire, dans... les orphelinats, les asiles, les crèches. Les premiers cas sont lents à découvrir. Quand on s'avise de leur existence, un tiers de l'école est contaminé... J'ai vu... des épidémies de cinquante, quatre-vingts cas, se constituer sous mes yeux en quelques semaines. » Dans notre observation, c'est à peine, dans l'espace de cinq mois, un trentième de l'effectif total de l'école maternelle qui est atteint.

c) L'origine raciale des enfants atteints est remarquable : un enfant sur deux est d'origine noire, ce qui contraste singulièrement avec la proportion réelle d'enfants noirs par rapport aux enfants blancs dans cette école. Les enfants noirs sont donc atteints avec prédilection, même lorsqu'ils sont nés en France (Serge T...). Basset note que, au Sénégal, la fréquence des affections à $M$. audouini chəz les européens est infime par rapport à ce qu'elle est chez les noirs. On peut ainsi postuler soit une sensibilité plus grande de la race noire à $M$. audouini (sens. lat.) (mais ce n'est pas l'avis de Basset), soit une facilité plus grande à s'infecter, du fait d'un ensemble de raisons qui tiennent autant à l'éthologie qu'à l'hygiène.

Les noirs de notre enquête sont d'origine diverse (Cameroun, Sénégal, Gabon). Ce sont autant de pays où il existe une forte endémie de $M$. audouini (sens. lat.).

(1) Peut-être, en fait, la diffusion par mode épidémique est-elle plus limitée qu'il n'apparaît de prime abord, la source de contamination originelle pouvant être multiple: un certain nombre des enfants noirs impliqués ici ont pu arriver en France déjà atteints de teigne. 
Aussi nous semble-t-il raisonnable de penser que ce sont les enfants noirs qui ont introduit la teigne dans cette école, plus spécialement ceux dont la date d'arrivée en France est récente (pour 1971, les petites Di..., en France depuis octobre 1970 ; pour 1972, la petite A..., arrivée en France en mars 1971).

Il semble donc que nous soyons en présence de deux petites épidémies à évolution lente, dues à $M$. audouini et d'origine africaine.

d) Caractéristiques des souches: Vanbreuseghem, dès 1950, a établi une distinction entre l'Audouini africain et l'Audouini européen et a fait du champignon africain une espèce nouvelle: Sabouraudites (Microsporum) langeroni, ce sur des critères d'ordre clinique $(M$. langeroni détermine plus fréquemment des lésions d'herpès circiné que $M$. audouini) et biologique (critère de pathogénicité pour le cobaye : $M$. langeroni est le plus souvent inoculable au cobaye, alors que $M$. audouini ne l'est pas).

Nos souches, toutes semblables, ont donné des colonies à développement assez lent, à duvet ras, de couleur beige rosé ; leur revers était coloré, mais la couleur n'était pas exactement celle qui a été signalée par Vanbreuseghem dans ses descriptions (ce revers était ocre rouge, mais d'une teinte plus foncée au centre et se dégradant vers la périphérie, au lieu de la teinte groseille traditionnelle).

La culture sur Sabouraud ordinaire ne nous a pas permis d'obtenir des fuseaux, mais seulement de très volumineuses chlamydospores.

L'inoculation au cobaye a été positive pour la plupart des souches (sur cinq souches inoculées (2), quatre se sont révélées positives; elles émanent aussi bien des enfants de race blanche que des enfants de race noire).

Ainsi, comme nous pouvons déjà en avoir déjà la présomption de par les critères mêmes de l'épidémie et la clinique elle-même (existence d'herpès circinés), nos souches peuvent donc être rapportées à Sabouraudites langeroni.

Ainsi donc, il n'est pas sans intérêt de constater qu'il puisse exister en France de petites épidémies dues à $M$. langeroni. Ces épidémies, du fait de l'inadaptation du champignon à nos climats et à des conditions d'hygiène satisfaisantes, seraient d'évolution relativement lente, mais elles pourraient affecter, en France même, non seulement les enfants de race noire, mais également les enfants de race blanche. Il est également notable que ces épidémies se soient produites chez des enfants en bas âge (moins de sept ans), fait noté déjà par Juminer pour le Sénégal.

Insister sur cette allure un peu spéciale de l'épidémie, n'est-ce pas mettre en avant un nouveau critère de différenciation entre $M$. audouini et $M$. langeroni (en Afrique noire, on ne peut guère étudier le mode épidémique à cause de l'endémie teigneuse persistante), et donner un argument supplémentaire à la distinction proposée par Vanbreuseghem ? Le mode évolutif des épidémies dues à $M$. langeroni, en dehors des zones d'endémie, lui serait propre (3).

(2) Nous remercions bien vivement le $\mathrm{D}^{\mathrm{r}}$ de Vroey (service du $\mathrm{P}^{r}$ Vanbreuseghem) qui a, par cette technique d'inoculation, prouvé la nature réelle de la première de nos souches.

(3) L'épidémie relatée par Coudert et al., en 1964, a évolué de façon toute différente. Bien que l'origine de cette épidémie soit également un enfant d'Afrique noire, il ne nous est pas dit si le dermatophyte en cause était $M$. audouini sensu stricto ou $M$. langeroni: or les deux champignons semblent coexister en Atrique. 


\section{B) Les modalités de contagion.}

En ce qui concerne la transmission, elle nous semble scolaire plus que familiale, bien que ce deuxième mode de contagion puisse intervenir de façon certaine (c'est le cas de deux petits français, B... Alain et son frère Jean-Louis, qui fréquentent, l'un l'école maternelle, fortement contaminée, l'autre l'école primaire, où aucun autre cas ne s'est déclaré). Le tableau 2 permet de comparer les incidences (familiale et scolaire) de l'affection.

\section{Tableau II}

Liens scolaires et familiaux des enfants contaminés

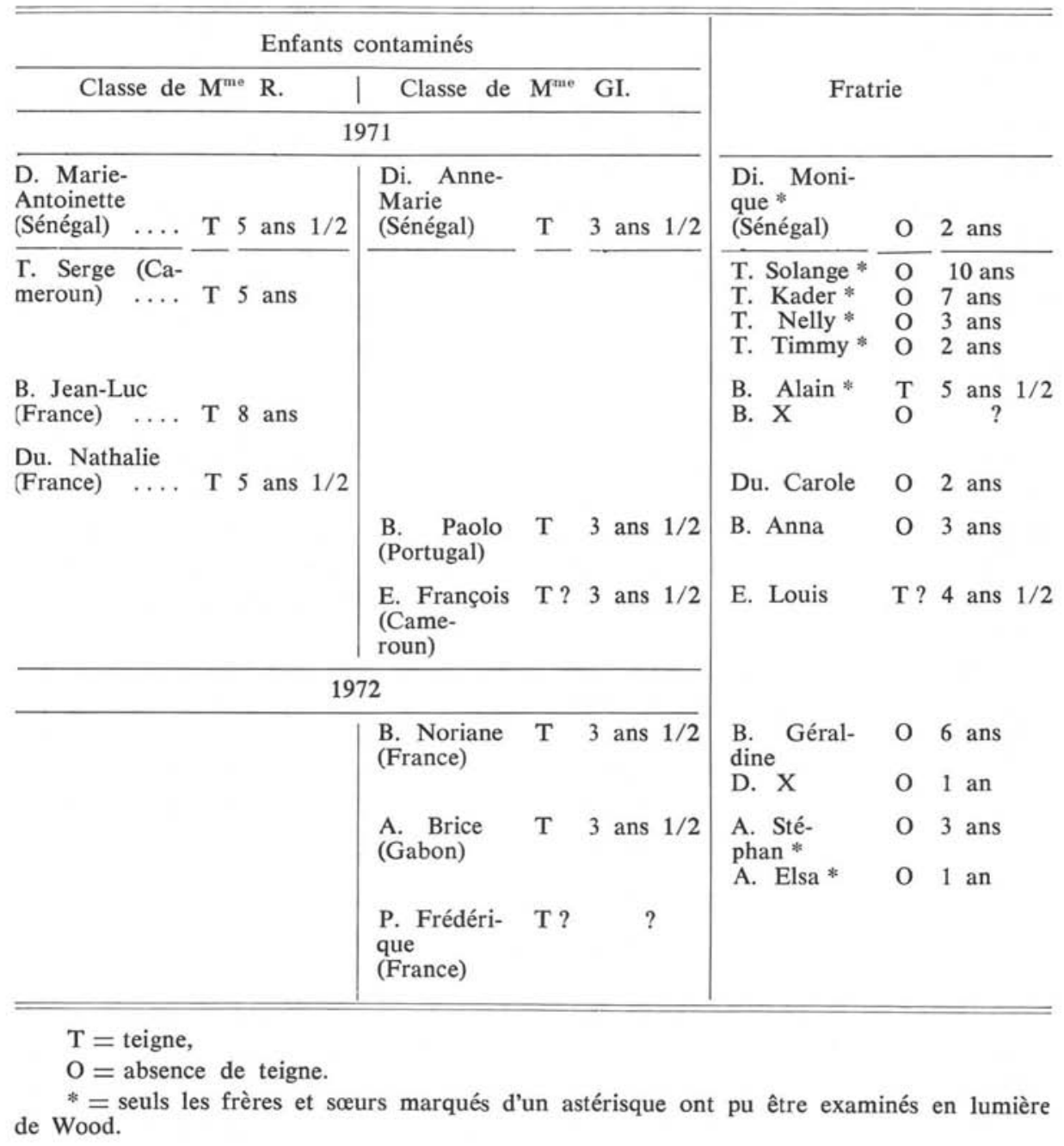


Les enfants teigneux comptent en tout quatorze frères et sœurs. Deux fois, la contamination familiale est possible, mais, dans un cas, la contamination peut être tout aussi bien scolaire. L'incidence familiale semble donc faible. même :

Nous avons tenté d'élucider certains mécanismes de transmission dans l'école

a) Nous avons d'abord essayé de préciser s'il existait ou non des porteurs sains : malheureusement, cette enquête n'a pu être réalisée qu'une quinzaine de jours après l'éviction des petits teigneux. Avec des fragments de moquette stérile, nous avons, suivant la technique de Mariat, brossé le cuir chevelu des enfants dans les deux classes les plus atteintes, et cela même à leur place habituelle dans la salle de classe. Puis il a été procédé à l'ensemencement sur milieu de Sabouraud additionné d'actidione : aucune colonie ne s'est développée.

b) Une tentative a été faite ensuite pour retrouver des spores dans le milieu extérieur: une vingtaine de portemanteaux non individuels ont été passés à la moquette. Aucune colonie ne s'est développée.

c) Les plus jeunes enfants étant soumis à la sieste quotidienne, nous avons cherché des spores sur les oreillers des petits teigneux: nous avons ainsi obtenu plusieurs colonies de $M$. langeroni. Cependant, les couchettes étant strictement individuelles et le linge correctement bouilli, la contamination ne peut pas s'effectuer par les oreillers.

\section{C) Les raisons sociologiques.}

Que ces petites épidémies, avec leur caractère très particulier, aient pu éclater dans l'école en question, cela ne nous semble pas le fait du hasard. Cela nous paraît dû, très exactement, à l'importance numérique des noirs dans ce quartier de la ville (plus de quinze ménages totalisant quarante-six enfants). Alors que les autres écoles de la ville ne comptent que très peu d'enfants originaires d'Afrique noire (au maximum un ou deux), l'école où nous avons détecté nos cas en héberge souvent plusieurs par classe.

On comprend qu'il puisse exister, de surcroît, de larges possibilités de contact et donc de contamination entre les familles, souvent de même ethnie et qui ont ainsi tendance à rechercher une vie semi-communautaire : cela pourrait contribuer à faire naître une endémie (surtout chez les enfants en bas âge qui ne fréquentent pas encore la maternelle). Le fait qu'un nouveau contingent de noirs vient s'ajouter, chaque année, sans avoir subi d'examen mycologique, multiplie encore ce danger.

\section{Déductions prophylactiques}

Rappelons d'abord une vérité élémentaire: l'expérience montre qu'un certain nombre de cas de teignes scolaires sont diagnostiqués et soignés silencieusement, c'est-à-dire qu'ils ne font l'objet d'aucune déclaration. La teigne ne faisant, trop souvent, l'objet d'aucun prélèvement, on ne connaît pas le dermatophyte en cause. 
Or si cela peut paraître moins grave pour les cas de teignes à $M$. canis (de contamination principalement animale, encore que des cas de contamination interhumaine aient été décrits), il peut être désastreux de laisser évoluer une épidémie de $M$. audouini (même si « le génie épidémique » de $M$. langeroni paraît moindre, en France, que celui de l'Audouini typique). D'où la nécessité d'une enquête avec les moyens appropriés (la lampe de Wood et la culture avec identification précise de l'espèce en cause), menée rapidement par un organisme qualifié, chaque fois que plusieurs cas sont signalés dans une école.

Il nous paraît nécessaire, en outre, de soumettre tout enfant noir, à son admission à l'école, en début d'année scolaire, à un examen mycologique précis.

Enfin, il nous paraîtrait judicieux, pour éliminer toute possibilité d'endémie teigneuse, d'examiner chaque année la totalité des membres de la petite collectivité noire.

\section{Bibliographie}

Badillet (G.), Pringuet (R.), Demay-Wechsler, 1972. - Notions récentes sur les Microspories dans la région parisienne. C.R. $14^{e}$ cong. int. Derm. (Padoue-Venise).

BASSET (A.) et BASSET (M.), 1959. - Les teignes du cuir chevelu à Dakar. Bull. soc. franç. Derm. Syph., 66, 474-476.

—, —, 1960. — Les teignes du cuir chevelu au Sénégal. Enquête préliminaire. Bull. soc. path. exot., 53, 500-505.

—, - , 1961. - Les teignes du cuir chevelu et les épidermomycoses à Dakar. Enquête. Bull. soc. path. exot., 54, 810-816.

-, —, 1964. - Fpidémiologie des teignes. Mycopathol. et Mycol. appl., 24, 233-242.

Colomb (D.), Battesti (M. R.), Lu (H. T.), Novion (M.), 1972. - Les mycoses cutanées dans la région lyonnaise. Analyse de 500 prélèvements sur milieu de Sabouraud pratiqués sur des dermatoses suspectes d'une étiologie mycosique. Lyon Médical, 227, 703-708.

Coudert (J.), 1964. - Répartition géographique des dermatophytes en Europe. Ann. soc. belge Méd. trop., 44, 725-740.

-, Battesti (M. R.), Despeignes (J.), Michel-Brun (J.), 1964. - Remarques sur une épidémie de teigne à $S$. audouini dans une collectivité d'enfants. Prophylaxie et traitement. Bull. soc. franç. Derm. Syph., 71, 115-119.

Degos (R.), Bernadou (M.), VACHON (M.), 1971. - Teignes du chevelu observées à l'hôpital Saint-Louis de 1967 à 1971. C.R. 5 $5^{\circ}$ cong. int. Myc. hum. et an., 96-97.

Gilot (B.), Amblard (P.), Groullier (A.), Dugois (P.), 1970. - Les mycoses cutanées au Centre Hospitalier de Grenoble. Premier bilan. Grenoble Médico-chirurgical, 8, 397407.

-, Dugois (P.), Amblard (P.), Imbert (R.), Vigny (F.), 1970. - Etude statistique des dermatophytes dans le Dauphiné du Nord. Bull. soc. franç. Derm. Syph., 77, 182-185.

Juminer (B.), 1968. - Etude écologique et clinique des dermatophytoses dépistées au C.H.U. de Dakar. Arch. Inst. Pasteur Tunis, 45, 168-175. 
-, Diallo (S.), Diagne (S.), 1969. - Les teignes scolaires en Basse-Casamance. Arch. Inst. Pasteur Tunis, 46, 205-216.

Sabouraud (R.), 1910. - Les teignes. Masson et $C^{i e}$, édit., Paris.

VANBreuseghem (R.), 1950. - Contribution à l'étude des Dermatophytes du Congo Belge : le Sabouraudites (Microsporum) langeroni n.sp. Ann. Parasit. hum. comp., 25, 509-517.

-, 1962. - Faut-il distinguer Microsporum langeroni de Microsporum audouini ? Bull. soc. franc. Derm. Syph., 70, 34-37. 